\title{
XLIX. On the pressure of the electric wind in hydrogen containing traces of oxygen
}

\section{A.P. Chattock \& A.M. Tyndall B.Sc.}

To cite this article: A.P. Chattock \& A.M. Tyndall B.Sc. (1910) XLIX. On the pressure of the electric wind in hydrogen containing traces of oxygen, Philosophical Magazine Series 6 , 19:112, 449-460, DOI: $10.1080 / 14786440408636824$

To link to this article: http://dx.doi.org/10.1080/14786440408636824

曲 Published online: 21 Apr 2009.

Submit your article to this journal $\lceil\pi$

Џ Article views: 6

Q View related articles $\square$

Citing articles: 3 View citing articles 5 
THE

LONDON, EDINBURGH, AND DUBLIN

PHILOSOPHICAL MAGAZINE

\author{
AND \\ JOURNAL OF SCIENCE.
}

[SIXTH SERIES.]

APRIL 1910.

XLIX. On the Pressure of the Electric Wind in Hydrogen containing traces of Oxygen. By A. P. СенатTock, Professor of Physics, and A. M. TYNDalu, B.Sc., Lecturer in Physics, in the University of Bristol*.

TN a recent papert dealing with the influence of oxygen 1 on point-discharge in hydrogen at atmospheric pressure the present authors showed that if the amount of oxygen in the hydrogen be reduced to a fraction of one per cent., certain phenomena accompanying the negative discharge are modified in a marked way.

Among these it seemed possible that the velocity of the negative ions was to be classed, its value being apparently raised as the hydrogen approached a state of purity (loc. cit. pp. 44-47). As, however, the principle upon which this statement was based is not above criticism (see below p. 456) it seemed desirable to measure the ionic velocities again in some other way.

This we have now done, using the wind-pressure method $\ddagger$ for the purpose, and the results obtained are discussed in what follows.

* Communicated by the Authors.

† Phil. Mag. 1908 [6] vol. xvi. p. 24.

$\ddagger$ Phil. Mag. 1899 [5] vol. xlviif. p. 401.

Phil. Mag. S. 6. Vol. 19. No. 112. April 1910. $2 \mathrm{G}$ 


\section{Method of the Experiments.}

The hydrogen and its admixture of oxygen were prepared and introduced in the manner described in our paper of 1908 (p. 30), the identical apparatus being used for the purpose. As the arrangement of the discharge-vessels and manometer required considerable alteration they were constructed afresh.

Fig. 1 gives a perspective view (diagrammatic) of the new design.

Fig. 1.

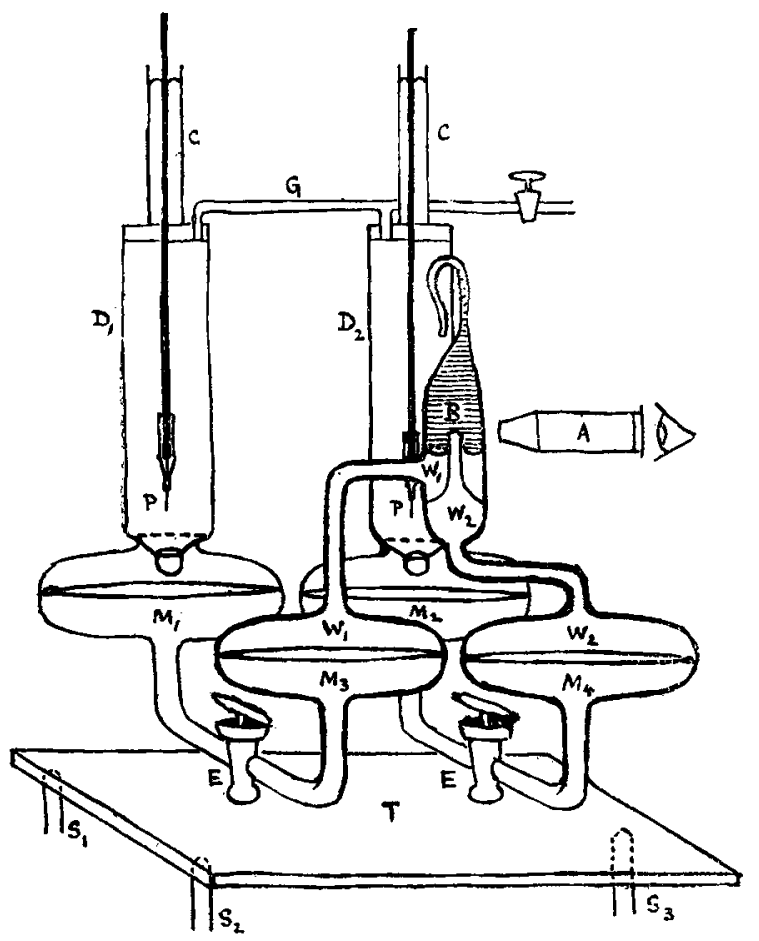

$T$ is a tilting-table, supported on three points $S_{1} S_{2} S_{3}$, and $\mathrm{S}_{3}$ is the end of a spherometer screw, on the large divided head of which (not shown) it is possible to estimate a travel of $S_{3}$ of $1 / 40000$ inch.

Upon $T$ is fixed the glass discharge apparatus. The four bulbs $M$, each $10 \mathrm{~cm}$. in diameter, contain mercury in their 
lower halves. Above $M_{1} M_{2}$ are the discharge-tubes $D_{1} D_{2}$ containing the gas to be experimented on. Above $\mathrm{M}_{3} \mathrm{M}_{4}$ is water $W_{1} W_{2}$.

$\mathrm{W}_{2}$ is continued upwards to form a bubble of water, $\mathrm{B}$, in benzene (shaded) at the top of an inverted glass funnel sealed into the tube which contains it. The upper surface of the bubble is focussed on to the cross-wire of the microscope A, and forms a very delicate indicator of the small differences of pressure applied botween the surfaces of $M_{1}$ and $M_{2}$. The diameter of the bubble is about $4 \mathrm{~mm}$.

In making a measurement the bubble is kept on the crosswire by tilting $T$ with $S_{3}$, and the tilt then affords a measure of the difference of pressure applied*.

When working well the movement of the bubble is just visible under a pressure difference of $10^{-2}$ dyne $\mathrm{cm} .^{-2}$

$\mathrm{E} \mathrm{E}$ are taps for locking the manometer during the pumping out of the discharge-tubes.

At the bottom of each discharge-tube is a plate of thin rolled sheet zinc covered with perforations (about 500 of them) and connected with a sensitive galvanometer. This plate, shown by a dotted line, is the surface against which the point $P$ discharges. It is more convenient than the ring used in the earlier wind-pressure work, as it does not entail so long a discharge-tube. The perforations must be such that the pressure below the plate is the average of the pressures on its upper surface. For this to occur the perforations must all be equal and equally spaced, and the flow of gas (if any) through the plate at each perforation must be proportional to the difference of pressure producing it.

This is not an easy condition to fulfil in general; but in our case the differences of pressure that could occur between the two sides of the plate were so extremely small that we were able to obtain the requisite proportionality with sufficient accuracy by using the point of a fine sewing-needle suitably supported to make the perforations.

* This manometer is a modification of the one designed twelve years ago for the original measurements of the electric wind (Chattock, Phil. Mag. 1899, loc. cit.). The latter was subsequently improved by Mr. J. D. Fry, and in this form used and described by Dr. T. E. Stanton (Proc. Inst. C. E. vol. clvi. 1903-4, pt. ii.). In the instrument just described benzene replaces oil as it is less sluggish, and its surface remains clean for much longer periods, provided that the water in contact with the benzene is air free. When, as in our case, it is necessary to trap the water with mercury the effect of the latter on the working of the manometer can be practically got rid of by using large mercury surfaces and putting the mercury tubes at right angles to those containing the water, as indicated in the diagran. The manometer must then bis calibrated in terms of a second manometer containing water only. 


\section{Prof. Chattock and Mr. Tyndall on Pressure of the}

The difficulty that the plate could not be perforated right up to the side of the discharge-tube was met by putting extra holes as near the edge as possible.

$D_{1}$ and $D_{2}$ are connected by the tube $G$; and the gas is introduced through $G$ by way of a glass spiral (not shown) sufficiently slender to admit of the tilting of the manometer.

The discharging point $P$, of finest platinum wire cut obliquely with sharp scissors, and sheathed almost to its end with glass, is supported by a thin steel rod sliding in a mercury-tight stuffing-box. This rod is so protected by a column of mercury, $C$, that no part of its surface which has been exposed to the air passes into the discharge-vessel when the point is lowered.

Stuffing-box and connecting tube, $G$, are attached to an ebonite plug which closes the top of $\mathrm{D}$; the joint with $\mathrm{D}$ being made gas tight by an indiarubber band (not shown) protected from the air by mercury.

Below the perforated plate hangs a small tube of phosphorus pentoxide.

The internal diameter of $\mathrm{D}_{2}$, the tube we chiefly used, is $4.47 \mathrm{~cm}$. One turn of the tilting-serew corresponds with a pressure of 9.07 dyne-cm. ${ }^{-2}$ The pressure of the gas was adjusted to $75 \mathrm{~cm}$. The temperature was that of the room.

The principle of the wind-pressure method may thus be described. If a point discharges a current of strength $c$ through a uniform tube of cross-section $a$ against a plate at a distance $z$ from the point, the specific velocity of the ions $\mathrm{V}$ is given by the expression

$$
\mathrm{V}=\frac{c}{a} / \frac{d p}{d z}
$$

where $p$ is the average pressure of the electric wind per unit area of the plate; it being assumed that the plate fills the tube, and that ions of one sign only are present in the distance $d z$.

The measurements were made in the same way as those described in the 1899 paper (loc. cit.).

\section{Discussion of Results.}

Earlier measurements by the wind-pressure method * led to values of $\mathrm{V}$, which are in satisfactory agreement with those obtained by other methods. For dry hydrogen the values of $\mathrm{V}+$ and $\mathrm{V}$ - were respectively $5 \cdot 4$ and $7 \cdot 43 \mathrm{~cm}$.sec. ${ }^{-1}$ in a field of 1 volt-cm..$^{-1}$, the hydrogen containing a

$$
\text { * Phil. Mag. } 1901 \text { [6] vol. i. p. } 79 .
$$


Electric Wind in Hydrogen containing traces of Oxygen. 453

small but unknown quantity of air. Other methods gave for dry hydrogen $5 \cdot 67$ and $7 \cdot 18$ as the most probable values of $\mathrm{V}+$ and $\mathrm{V}-*$.

In the present experiments we found that when more than, say, 0.5 per cent. of oxygen is present in the hydrogen, the values of $\mathrm{V}$ by the wind-pressure method are about the same as before $(\mathrm{V}+=5 \cdot 8, \mathrm{~V}-=7 \cdot 6)$; but in the case of negative discharge alone, a very striking decrease in the values of $d p / d z$, i. e. increase in the apparent values of $\mathrm{V}-$, is observed as the hydrogen is made purer, the increase being more than forty-fold in the purest gas we could at first obtain. This is shown in the Table of Results, where are collected the bulk of our determinations; and graphically in Curves I. (p. 454), in which the first and third columns of the Table are plotted together.

Except where otherwise stated all measurements are given in c.G.S. units.

Table of Results.

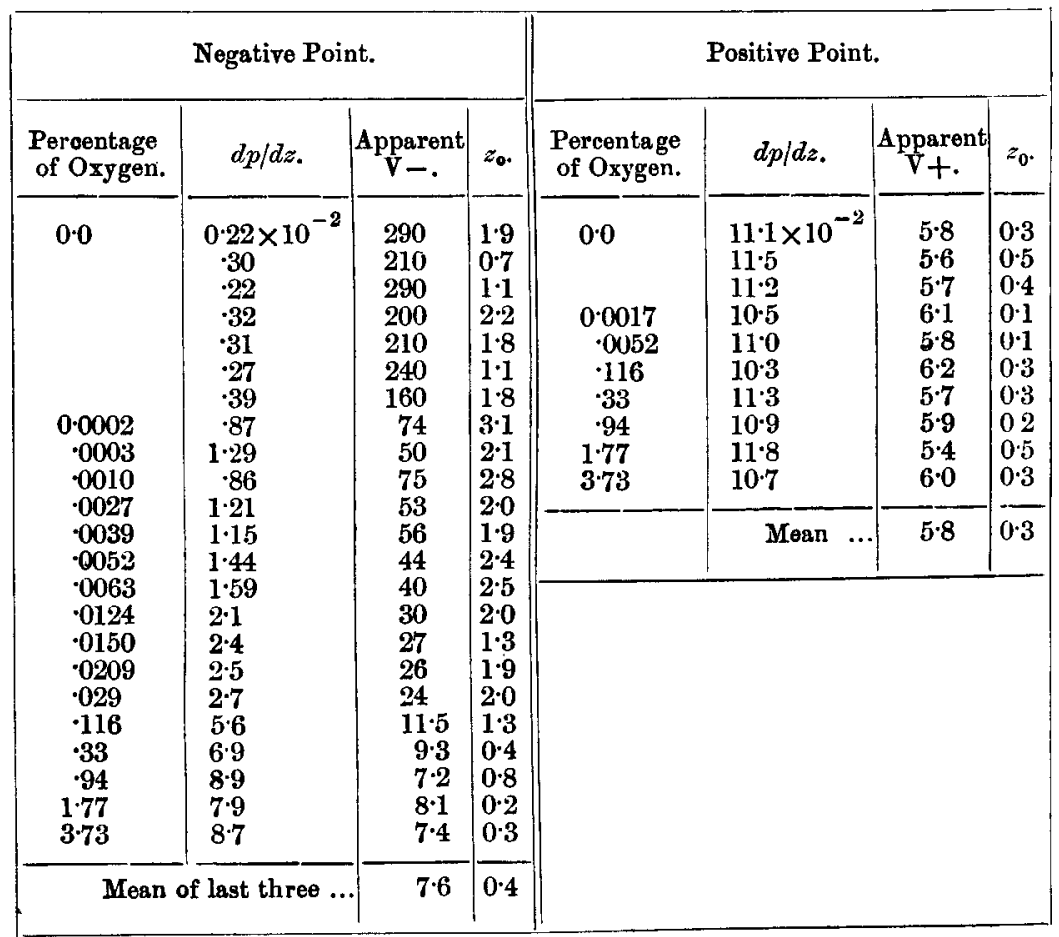

* Winkelmann, Handbuch der Physik, iv. p. 555. 
454 Prof. Chattock and Mr. Tyndall on Pressure of the

$d p / d z$ is the slope of pressure for a current of 1 microampere from the point, and $V$ is the ionic velocity in a field of 1 volt-cm. ${ }^{-1}$

Curves I.

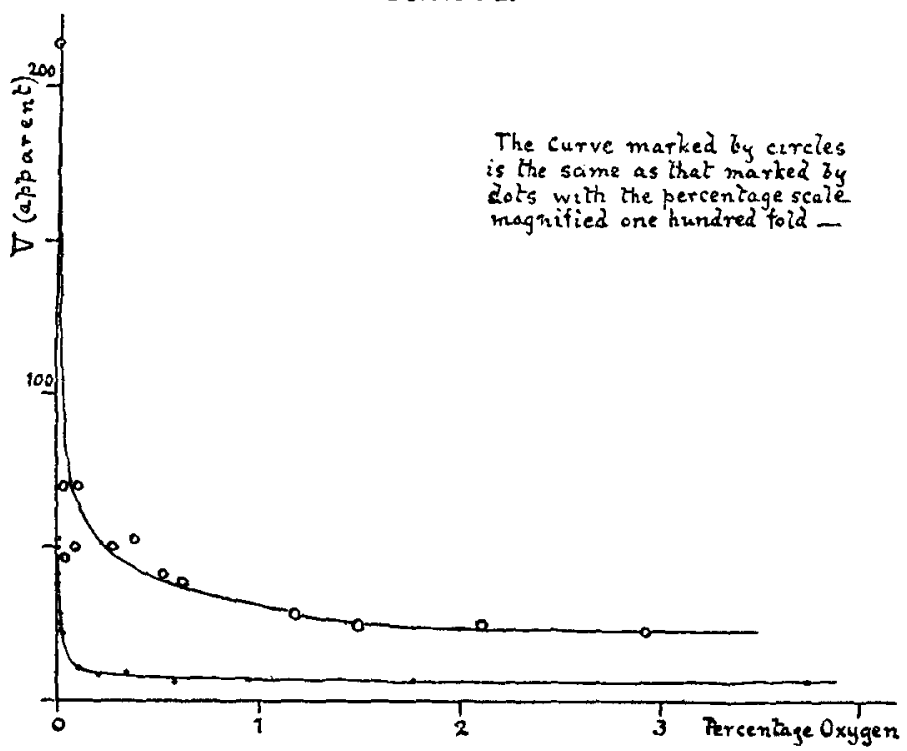

It was found that the values of $d p / d z$ were not independent of the current nsed to obtain them, and that they increased as the current was reduced-the negatives considerably. The current used for all but the very low percentages was about 2 microamperes. For these, in the case of negative discharge only, it was increased about three-fold, as the pressures to be measured were then so very low.

The numbers in the second column have all been corrected to zero current. This meant an increase in the pressure slope per microampere obtained from a current of 2 microamperes of abont 20 per cent. for negative and 5 per cent. for positive discharge, in the case of the higher percentages.

Now it is most unlikely that the negative ion travels at anything like the speeds shown here-speeds which are moreover out of all proportion greater than those suggested by our 1908 work; and, in fact, we found later that it was possible to obtain values of $d p / d z$ which were actually though very slightly negative for discharge in what was perhaps still purer hydrogen*.

* A little paraffin-wax had been discovered between the two sets of experimente upon the glass under one of the mercury seals; and this may have given riae to a very slow leak of air into the apparatus between the wax and the glass in the first experiments. 
This result proves that here at any rate we have a case of discharge which cannot consist of the simple stream trom point to plate of ions of like sign to the point postulated for the formula. It strongly suggests the presence of positive ions travelling against the wind, ions which, even if present in smaller numbers than those from the point, might still reverse the pressure as they travel slower; and it is then of course only reasonable to regard these ions as the cause also of the lowering of $d p / d z$ in those samples of hydrogen which contained oxygen, and for which $d p / d z$ was not negative but only very small.

The view that back discharge is the cause of the reduction of the wind is further borne out by the appearance of the plate when examined in the dark. In the purest hydrogen, and for a negative point, the whole plate glows brightly over the surface presented to the point; but the glow fades gradually as small quantities of oxygen are added to the gas. At a current of 2 microamperes, for instance, the plate was still faintly luminous with $0 \cdot 1$ per cent. of oxygen present, but became quite dark before the percentage reached 0.9 .

With a positive point no such effect is observed. At 2 microamperes the plate was quite dark, but a very faint luminosity was always obtainable with much stronger currents which was apparently little if at all affected by the amount of oxygen present.

It thus appears that for negative discharge the glow is a maximum when $d p / d z$ is lowest, viz. for the purest hydrogen, and that as oxygen is added the glow fades and $d p / d z$ increases, until at about the point where $d p / d z$ gives normal values of $\mathrm{V}$ - the glow vanishes.

If, then, we may take the glow to mean ionization at the plate, we have in these facts strong support for the view that the lowering of $d p / d z$ is due to back discharge.

It is interesting that under certain conditions the glow seems to become unstable, tending to contract suddenly often to a point which glows brightly, and is probably coincident with some slight projection on the plate. For if at any region on the plate the back discharge accidentally increased, the lines of force from the point would converge towards that region and concentrate the current there, thus tending to increase the back discharge still further, and so to intensify the concentration of the lines. We have seen this effect on several occasions, and there can be little doubt that it has been responsible for a certain irregulurity which characterizes the wind of negative discharge. And this suggests another consideration. As the point is moved further from the plate 


\section{Prof. Chattock and Mr. Tyndall on Pressure of the}

the field at the surface of. the plate is increased, owing to the increased quantity of free electricity in the discharge-tube. It may well be that this entails an increase in the back discharge when the latter is present, and a consequent further decrease in the wind pressure. If this is so the apparent increase in $\mathrm{V}$-, while directly dependent upon back discharge alone, may be indirectly dependent through it upon two distinct causes : one the cause of the back discharge itself, and the other the cause, just referred to, which renders the back discharge greater at large than at small values of $z$.

In the 1901 work on hydrogen large variations were met with in the calculated values of $\mathrm{V}$ - which could not be attributed to errors of observation, and which seemed to imply real changes in $\mathrm{V}$ - . At the time it was suggested that the intermittent escape from the point of occluded gases other than hydrogen might be responsible for the effect. In the light of the present experiments, however, it is clear that changes in the back discharge due to very small changes in the amount of oxygen present are capable of having produced even larger changes in the value of $V$ - than those which were actually found. As such small changes in the purity of the hydrogen must certainly have occurred in this early work, it seems safe to replace occluded gas by variation of back discharge in explaining the variations of $\mathrm{V}-$.

\section{Heating Effect of Discharge.}

In our 1908 paper it was shown that if point discharge occurs in a closed vessel the rise of temperature of the gas depends on the specific velocity of the ions; the rise per watt of electrical energy supplied being a simple function of $V$ and increasing with it.

The meaning of this is that if $\mathrm{V}$ be increased from any cause, the ions by passing through the gas more rapidly will exist at any moment in smaller numbers between the point and the plate. The drag on the gas and consequently the wind will be less. Hence the cooling will be less, and a higher final temperature will be reached.

Abnormally high temperature rises were recorded in the case of negative discharge through nearly pure hydrogen (loc. cit. pp. 44-47); and this was the reason we thought, as mentioned above, that $\mathrm{V}$ - might show a corresponding increase above its value in less pure gas.

But back discharge will also lead to rise of temperature by reducing the wind, and we now find that a reduction of the oxygen present promotes back discharge when the point is 
Electric Wind in Hydrogen containing traces of Oxygen. 457 negative. It is therefore much more likely that the high temperatures in question are due to increase in the back discharge rather than to any change in $\mathrm{V}$-.

$$
z_{0}
$$

There remains one other point to be referred to-the curious shifting of the negative wind-pressure curve parallel to itself-an effect which was first observed in the 1901 experiments.

In the present work it has been again met with, and now turns out to be another of the effects which a small amount of oxygen has the property of destroying. An examination of the wind-pressure curves themselves will make the matter clearer.

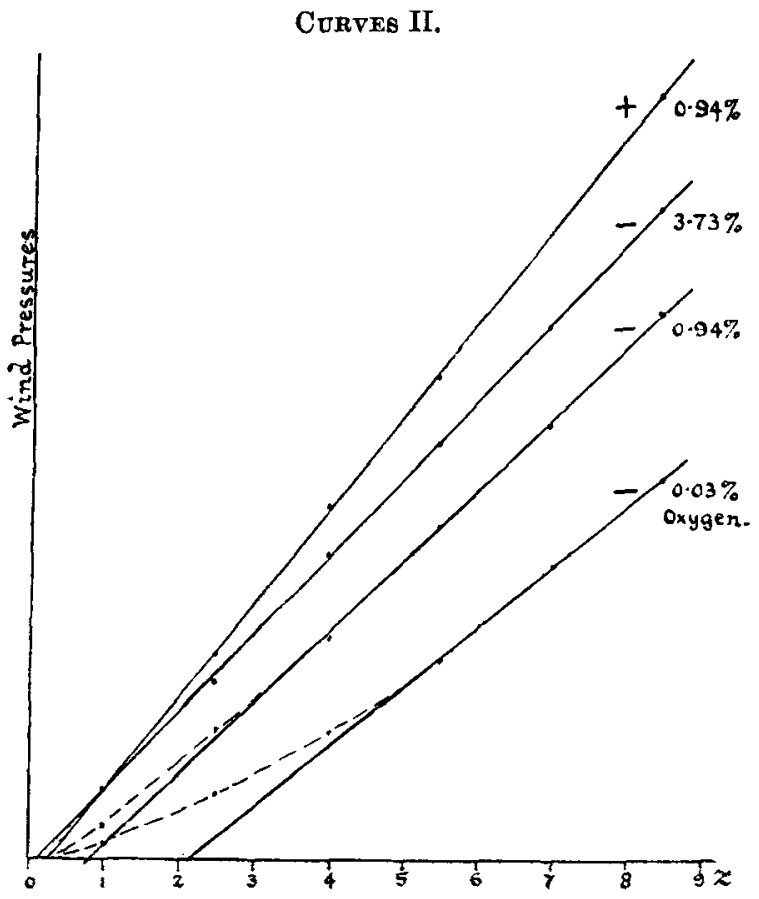

In Curves II. are given typical wind-pressure carves for positive and negative discharge, and a current of 2 microamperes in hydrogen containing various percentages of oxygen. The wind pressures for different distances, $z$, between point and plate are here plotted with $z$, the origin corresponding with the position of the plate. As it is the 


\section{Prof. Chattock and Mr. Tyndall on Pressure of the}

shape and position of each curve which is now of importance, we have arbitrarily chosen a different scale of ordinates for each so as to bring out these characteristics most clearly. The scale of abscissa is in centimetres, and is the same for all.

For positive discharge the curve is a straight line cutting the axis of $z$ very near the origin, and very little affected as regards shape or position by the presence of the moderate quantities of oxygen we used. The distance from the origin to the point of cutting is $z_{0}$ (see Table), and its value lies between 1 and $5 \mathrm{~mm}$.

For negative discharge the curve is also straight in its upper part, but in the case of low percentages of oxygen it bends towards the origin, so that $z_{0}$ which is now the cutting point of the straight part produced may have much higher values than for positive. Those we have observed have lain between 1 and $30 \mathrm{~mm}$.

In the curves the continuous lines are ruled straight, the dotted portions following the experimental points where necessary.

In the earlier experiments on hydrogen by the point and ring method (loc. cit. p. 87) the same sort of effects were met with; $z_{0}$ for negative varying between 8 and $13 \mathrm{~mm}$., and for positive between 0 and $2 \mathrm{~mm}$. The high value for negative was there attributed to a back discharge of positive ions from the ring, these being supposed to meet and combine near the ring with some of those from the point. In this way the small values of $d p / d z$ near the ring were explained, and the fact that beyond a certain distance from the ring the curve became straight was taken as proving that the combination all occurred within that distance.

This view is probably wrong. In the present experiments, for instance, the electricity in each cub. centim. of the discharge-tube was always considerably less than 1 E.S. unit, which means that for every ion there were more than $10^{9}$ molecules of hydrogen. The chances of back discharge ions combining with those from the point must thus have been negligibly small except quite close to the point, and they must be pictured as traversing the tube practically from end to end. Their effect on the wind-pressure curve will thus be to reduce its slope without altering its straightness; and so, unless we arbitrarily assume the existence of two kinds of positive ions, one of which combines with the negatives while the other does not, we find in back discharge a simple explanation of the abnormal ionic velocities, but not of the large values of $z_{0}$. 
There are two other ways in which these large values may possibly arise :-

(i.) The ions from the point may travel an appreciable distance before growing large enough to produce much wind.

(ii.) The gas may be ionized for an appreciable distance from the point-this being equivalent to a lengthening of the point so far as wind production is concerned.

It is possible that both these processes may be involved in the explanation of $z_{0}$. There is at any rate definite evidence available for (i.), since Franck has shown* that when discharge occurs in air from the surface of a fine wire in a strong field, it is extremely probable that the ions, whether positive or negative, do not reach their full size while travelling a distance comparable with $7 \mathrm{~mm}$. when driven by the strong fields he used.

Now if $z_{0}$ is to be due to (i.), we should expect its value in air to be less than $7 \mathrm{~mm}$. for the weaker fields which accompany discharge at a sharp point ; and in fact we find it to be about $3 \mathrm{~mm}$. for both positive and negative $\dagger$. This is consistent with Franck's result, and makes it likely that for air $z_{0}$ is what it is owing to the growth of the ions after leaving the point.

For negative discharge in hydrogen containing more than 1 per cent. of oxygen, and for positive discharge at all percentages, the mean value of $z_{0}$ is 3 to $4 \mathrm{~mm}$. It is possible that these numbers may be a little too low as the point (in the case of the hydrogen experiments only) unfortunately projected from its glass sheath rather further than it ought (about $3 \mathrm{~mm}$.), and there may have been a little discharge from its sides. The error cannot, however, amount to much more than a millimetre. Taking $z_{0}$ as 4 or $5 \mathrm{~mm}$., it is comparable with the value for air, and thus presumably explainable in the same way.

But for lower percentages of oxygen and negative discharge the case is different. $z_{0}$ is so many times larger that it appears necessary to invoke some special process to account for it.

The emission of corpuscles from the point seems to us to furnish such a process. It is known that oxygen tends to prevent this emission, and we now find that $z_{0}$ is only large when the amount of oxygen is small. If the removal of the

* J. Franck, Ann. der Phys. Vierte Folge, Bd. xxi. p. 984.

$\dagger$ This determination was mude with the present apparatus, in which the substitution of a plate for the ring renders dependable measurements of $z_{0}$ possible for the tirst time. 
oxygen is followed by a stream of corpuscles travelling at very high speeds from the negatively charged point, they may travel considerable distances before settling down into the state of ordinary negative wind-producing ions. Moreover they will ionize the gas at these considerable distances. Hence by the principles of both (i.) and (ii.) we may expect large values of $z_{0}$ on this view.

Experiments now in contemplation on the effect on $z_{0}$ of changes in the discharging point should throw light on this question.

In this connexion it is worth while to recall the fact that the state of the point has a very marked influence on the amount of combination which occurs between oxygen and hydrogen when the percentage of oxygen is low. For the four phenomena of glow, fall of wind pressure, shift of negative curve, and abnormally rapid combination all take place within about the same narrow limits of oxygen percentage; and it is therefore at least tempting to think that they may all ultimately prove traceable to a single source.

\section{Summary.}

1. The gradual removal of the last one per cent. of oxygen from hydrogen gives rise to a marked fall in the electric wind when a negative point discharges through the gas against a plate.

2. This fall is probably due to discharge of positive electricity from the plate, and may even amount to a slight reversal of the wind in very pure hydrogen.

3. The fall is accompanied by a glow upon the plate which may be regarded as further evidence for such back discharge.

4. Within the same limits of percentage change of oxygen the straight part of the negative wind pressure curves becomes shifted parallel to itself through a distance which may amount to 3 centimetres. It is suggested that this shift may be connected with the growth of the ions from the corpuscular magnitude to that of the ordinary negative ion.

5. None of the above effects occur with positive discharge in hydrogen, which is practically uninfluenced by the presence of oxygen up to the highest percentage (3.7) used.

In conclusion we wish to acknowledge our indebtedness to the Royal Society for a grant in aid of this research. 